\title{
Fibrinogen A $\alpha$ Thr312Ala polymorphism is associated with chronic thromboembolic pulmonary hypertension
}

\author{
J. Suntharalingam*, K. Goldsmith", V. van Marion\%, L. Long ${ }^{+}$, C.M. Treacy*, \\ F. Dudbridge $^{\#}$, M.R. Toshner*, J. Pepke-Zaba*, J.C.J. Eikenboom ${ }^{\star}$ and N.W. Morrell ${ }^{+}$
}

ABSTRACT: Although chronic thromboembolic pulmonary hypertension (CTEPH) is characterised by the persistence of organised thrombus, few pro-thrombotic risk factors have been identified in subjects with the disease. The aim of the present study was to compare the prevalence of eight functionally relevant haemostatic polymorphisms between CTEPH subjects and healthy controls.

Genomic DNA was isolated from 214 CTEPH subjects and 200 healthy controls, and analysed for Factor V Leiden, prothrombin guanine (G) to adenine (A) substitution at nucleotide 20210 $(20210 G>A)$, plasminogen activator inhibitor-1 4G/5G, tissue plasminogen activator 7351 cytosine (C) $>$ thymidine (T), Factor XIII 100G $>$, fibrinogen A $\alpha$ substitution of threonine with alanine at position 312 (Thr312Ala), fibrinogen $B \beta$ substitution of arginine with lysine at position 448 (Arg448Lys) and fibrinogen B $\beta$ 455G $>$ A polymorphisms.

A significant difference was demonstrated in fibrinogen A $\alpha$ Thr312Ala genotype and allele frequencies between CTEPH subjects and controls. The presence of the alanine allele significantly increased the risk of CTEPH.

The fibrinogen $A \alpha$ alanine $\mathbf{3 1 2}$ allele alters fibrinogen $\alpha-\alpha$ chain cross-linkage and has previously been associated with both increased risk of embolisation and increased resistance to thrombolysis. An association between this polymorphism and chronic thromboembolic pulmonary hypertension, therefore, supports an embolic aetiology for this disease, and may provide a mechanism by which thrombus persists following an acute event.

KEYWORDS: Coagulation, fibrinolysis, polymorphisms, pulmonary hypertension, thromboembolic

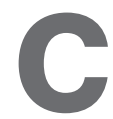
hronic thromboembolic pulmonary hypertension (CTEPH) is increasingly being recognised as an important cause of pulmonary hypertension, and is generally considered to represent an uncommon consequence of acute pulmonary embolic (PE) disease [1]. CTEPH has been reported to occur after $\sim 4 \%$ of cases of acute PE [2], whereas retrospective studies have described a preceding deep venous thrombosis (DVT) in approximately a third of cases [3, 4]. Therefore, it is presumed that, following an acute embolic event, thrombus persists and organises, resulting in chronic obstruction to pulmonary arterial blood flow.

Given the key role of thrombus formation in $\mathrm{CTEPH}$, it would intuitively be expected that there be some aberration of haemostasis, at either a local or systemic level. However, although hereditary thrombophilic conditions are commonly associated with acute PE and DVT $[5,6]$, no such conditions have been strongly linked with CTEPH [7]. Elevated serum levels of antiphospholipids and Factor VIII have been described previously [8, 9], but it is unclear whether these represent primary or secondary phenomena. As a result, no pro-thrombotic pathogenic mechanisms have been clearly shown to predispose to CTEPH. It is possible, however, that other steps in the haemostatic process, such as fibrin stabilisation or fibrinolysis, may be involved in the pathophysiology of the disease [10]. A number of single nucleotide polymorphisms (SNPs) have been described that predispose to arterial and venous thrombotic disease, by affecting these more global aspects of haemostasis [11-20]. This case-control study, therefore, uses a candidate gene approach to determine the potential relevance of these SNPs in CTEPH.

\section{AFFILIATIONS}

*Pulmonary Vascular Diseases Unit, Papworth Hospital, Papworth Everard ${ }^{\#}$ Medical Research Council Biostatistics Unit, and +University of Cambridge School of Clinical Medicine, Addenbrooke's Hospital, Cambridge, UK. "Dept of Haematology, Subdepartment of Thrombosis and Haemostasis, Leiden University Medical Centre, Leiden, The Netherlands.

CORRESPONDENCE

N.W. Morrell

University of Cambridge School of

Clinical Medicine

Addenbrooke's Hospital

Hills Road

Cambridge

CB2 2QQ

UK

Fax: 441223762007

E-mail: nwm23@cam.ac.uk

Received:

May 072007

Accepted after revision:

November 212007

STATEMENT OF INTEREST

None declared. 


\section{METHODS}

\section{Subjects}

All consecutive Caucasian patients attending Papworth Hospital (Papworth Everard, UK) with a diagnosis of CTEPH during 1999-2006 were offered entry to the present study. CTEPH was diagnosed through clinical evaluation, right heart catheterisation and appropriate imaging (chest radiography, ventilation/perfusion scintigraphy, computed tomographic pulmonary angiography, magnetic resonance pulmonary angiography and catheter-directed angiography), following a standardised protocol described elsewhere [4, 21]. CTEPH subjects $(n=214)$ included those with proximal $(n=169)$ and distal disease $(n=45)$. Healthy controls $(100$ males and 100 females) were recruited from a National Blood Service site in West London (UK) and comprised Caucasians aged 18-65 yrs.

The study protocol was approved by the Huntingdon Research Ethics Committee (UK). Written informed consent was obtained from each subject before entry into the study.

\section{Genotyping}

Genomic DNA was extracted from whole blood (Nucleon extraction kit; Amersham Biosciences, Little Chalfont, UK) and analysed for SNPs using commercially available primers (Taqman ${ }_{\circledR}$ SNP genotyping assay; Applied Biosystems, Foster City, CA, USA) using an ABI Prism ${ }^{\circledR}$ 7900HT Sequence Detection system (Applied Biosystems). Contract research services for genotyping were provided by Geneservice (Cambridge, UK). The eight SNPs studied were common genomic variations of the following haemostatic factors: Factor $\mathrm{V}$ (Factor V Leiden mutation or guanine $(\mathrm{G})$ to adenine $(\mathrm{A})$ substitution at nucleotide 1691 (1691G $>$ A); National Center for Biotechnology Information (Bethesda, MD, USA) SNP database accession code rs6025) [11]; prothrombin (20210G $>$ A; rs 1799963) [11]; plasminogen activator inhibitor-1 (4G/5G; rs1799768) [11]; tissue plasminogen activator (cytosine (C) to thymidine $(\mathrm{T})$ substitution at nucleotide $7351 \quad(7351 \mathrm{C}>\mathrm{T}$; rs2020918)) [17]; Factor XIII (100G>T; rs5985) [15]; fibrinogen $\mathrm{A} \alpha \mathrm{Thr} 312 \mathrm{Ala}$ (substitution of threonine (Thr) at position 312 with alanine (Ala); rs6050); and fibrinogen B $\beta$ Arg448Lys (substitution of arginine (Arg) with lysine (Lys) at position 448; rs4220); and fibrinogen B $\beta$ G455A (rs1800790) [22].

\section{Statistics}

Genotype and allele frequencies were calculated for each locus. Observed frequencies in controls were compared with those predicted by the Hardy-Weinberg equilibrium equation using the Chi-squared test. Linkage disequilibrium (LD) between SNPs was calculated from maximum likelihood haplotype frequencies estimated using the expectation maximisation algorithm [23].

Genotype and allele frequencies were compared between CTEPH and control subjects using the Chi-squared test, or Fisher's exact test in the case of low numbers. The risk of disease associated with each genotype (genotype model) was estimated by logistic regression analysis, where SNP genotype was coded in a three-level fashion, i.e. rarest homozygous genotype, heterozygous genotype and commonest homozygous genotype, with the latter being the reference category. For SNPs for which there were no rare homozygous subjects, the genotype was coded as heterozygous and compared with the most common homozygous genotype. The nature of the relationship between each SNP and risk of disease was also examined using models of dominant and recessive inheritance (allele model). In the dominant case, having one or more copies of the rare allele was compared with having no copies. In the recessive case, having two copies of the rare allele was compared with having one or no copies. Multiple logistic regression models of the effect of pairs of SNPs were used to assess whether or not there was any synergistic effect of having two SNPs. In view of sex differences between the two groups, all analyses were also repeated using multiple logistic regression models that adjusted for sex.

Adjustment for multiple tests was not performed for single SNP analyses, since each has previously been shown to be independently associated with an increased risk of thrombosis, and the present study aimed to re-address these hypotheses in the context of CTEPH. As such, calculations for each SNP constituted a separate and distinct analysis.

\section{RESULTS}

\section{Subjects and samples}

The baseline demographic characteristics of the CTEPH subjects are shown in table 1 . Only sex information was available for the 200 healthy controls, although it is known that they were Caucasian, aged 18-65 yrs and fulfilled the criteria required for blood donation.

The mean yield from SNP analyses was 96.3\%. All SNPs were in Hardy-Weinberg equilibrium, except for fibrinogen $B \beta$ Arg448Lys $(p=0.045)$. Only Fibrinogen B $\beta$ Arg448Lys and $455 \mathrm{G}>\mathrm{A}$ were demonstrated to be in $\mathrm{LD}\left(\mathrm{r}^{2}=0.91\right)$.

The genotype and allele frequencies of each of the SNPs in the two groups are shown in table 2.

\section{Polymorphism frequencies}

A significant difference was demonstrated in fibrinogen $A \alpha$ Thr312Ala genotype $(p=0.03)$ and allele $(p=0.01)$ frequencies between the CTEPH and control groups (table 2). The threelevel genotype model showed an association between the fibrinogen A $\alpha$ Thr312Ala SNP and CTEPH, although this did not reach significance for homozygotes (odds ratio (OR) 1.65, 95\% confidence interval (CI) 1.13-2.49 for heterozygotes, $\mathrm{p}=0.02$; OR 1.82, 95\% CI 0.92-3.61 for homozygotes, $\mathrm{p}=0.09$ ). A dominant model of inheritance of the Ala allele (i.e. one or

\begin{tabular}{llc} 
TABLE 1 & $\begin{array}{l}\text { Baseline demographics of chronic } \\
\text { thromboembolic pulmonary hypertension } \\
\text { subjects }\end{array}$ \\
Subjects $\mathbf{n}$ & 214 \\
Age yrs & $54.6 \pm 17.7$ \\
Male sex \% & 50.4 \\
Right atrial pressure $\mathbf{~} \mathbf{m} \mathbf{H g}$ & $9.3(6.2)$ \\
Pulmonary arterial pressure $\mathbf{~ m m H g}$ & $46.4 \pm 11.4$ \\
Cardiac index $\mathbf{L} \cdot \mathbf{m i n}^{-1} \cdot \mathbf{m}^{-2}$ & $2.1 \pm 0.6$ \\
Total pulmonary resistance $\mathbf{~ W U}$ & $12.8 \pm 5.2$ \\
\hline & \\
&
\end{tabular}


TABLE 2 Genotype and allele frequencies of the eight candidate genes

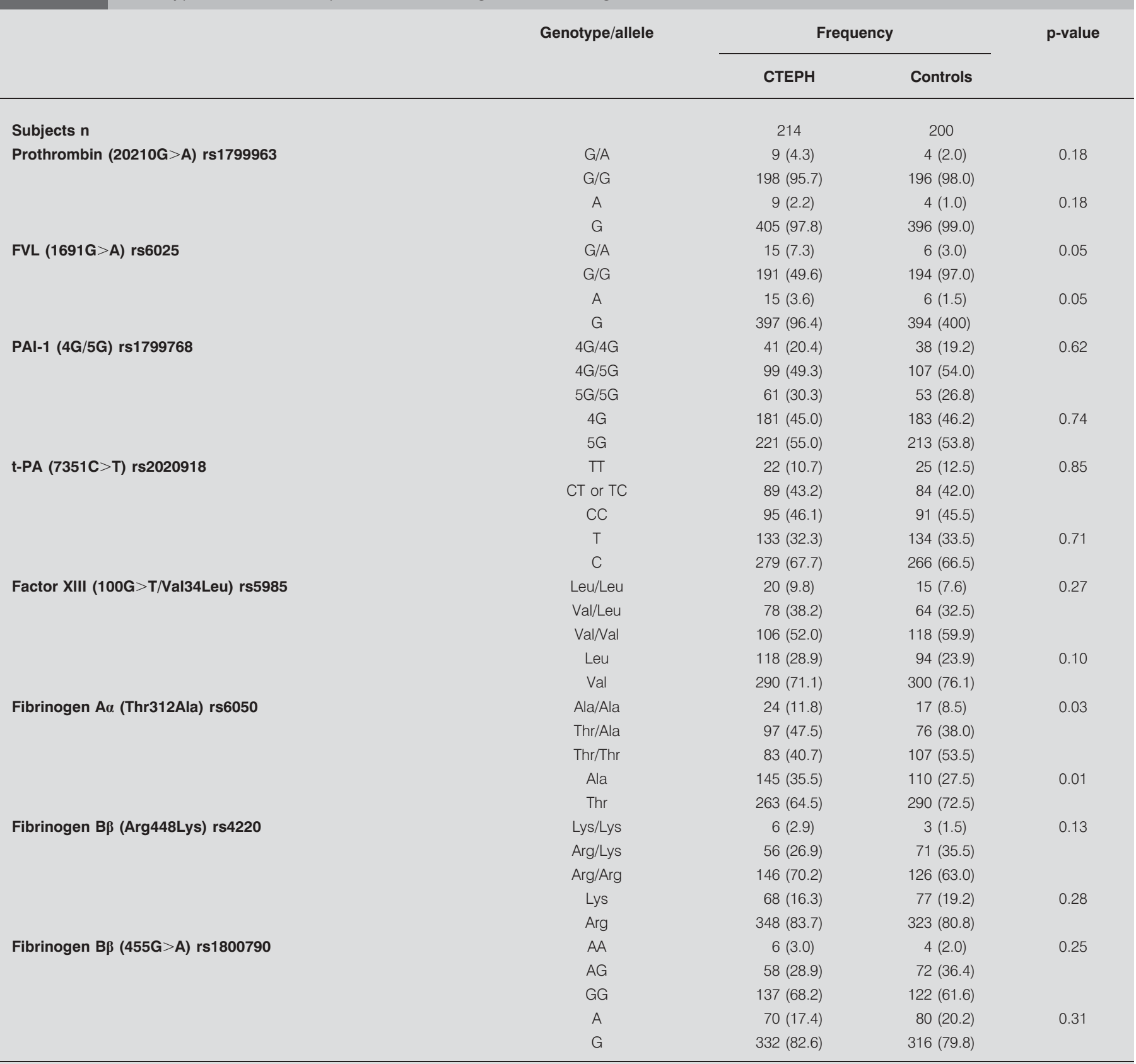

All frequencies are presented as $n(\%)$. CTEPH: chronic thromboembolic pulmonary hypertension; 20210G $>$ A: guanine (G) to adenine (A) substitution at nucleotide 20210; FVL: Factor V Leiden; PAl: plasminogen activator inhibitor; t-PA: tissue plasminogen activator; C: cytosine; T: thymidine; Val34Leu: substitution of valine (Val) 34 with leucine (Leu); Thr: threonine; Ala: alanine; Arg: arginine; Lys: Iysine.

two copies of the Ala allele versus none) showed increased odds of CTEPH (OR 1.68, 95\%CI 1.13-2.49, $\mathrm{p}=0.01$ ). There was no evidence of confounding of the relationship between the fibrinogen A $\alpha$ Thr312Ala SNP and CTEPH by sex (dominant model (females versus males): OR 1.06, 95\% CI 0.72-1.57, $\mathrm{p}=0.77$; three-level genotype model (females versus males); OR $1.06,95 \%$ CI $0.72-1.57, \mathrm{p}=0.78)$

There was a difference in Factor V Leiden genotype frequencies between the two groups, although this did not reach significance $(p=0.051)$. The Factor V Leiden mutation was associated with an increased risk of CTEPH (genotype model (no rare homozygotes): OR 2.54, 95\% CI 0.97-6.68, p=0.06), although, again, this was not significant.

There were no other significant differences between the two groups and no evidence of synergism between SNP pairs.

The six SNPs that failed to show an association with CTEPH were also used as a form of genomic control, to test for 
population stratification. The Kolmogorov-Smirnov goodnessof-fit test of the Chi-squared statistics for the association between each SNP and disease demonstrated that these followed a distribution that was not significantly different from a Chi-squared distribution $(p=0.35)$. These results suggest a lack of stratification.

\section{DISCUSSION}

The present study demonstrates that the presence of the fibrinogen A $\alpha$ chain Ala312 allele is associated with increased odds of being diagnosed with CTEPH. In addition, a significant association with the Factor $\mathrm{V}$ Leiden mutation was also noted. These are the first haemostatic polymorphisms to be reported in a large series of CTEPH subjects, and thus may add to understanding of the pathophysiological mechanisms behind this enigmatic disease.

Although CTEPH is widely considered to be a consequence of acute PE disease [1, 24], the evidence supporting this belief is largely circumstantial. One concern that has fuelled the debate over the aetiology of this disease is the discrepancy that exists between the thrombophilic profiles of DVT patients compared with CTEPH patients [25]. However, rather than viewing this as a barrier to the embolic theory of CTEPH, it is worth considering what might distinguish the minority of patients who develop CTEPH following a simple DVT from the vast majority who do not. In this context, it is important to consider not only the haemostatic factors that predispose to thrombus formation but also those that determine how thrombus is subsequently handled. A key factor in this process is clearly the fibrinogen molecule, which provides the essential building blocks for the formation of the fibrin clot. Abnormalities within this molecule may not only promote thrombus formation but also delay its degradation, thus encouraging the switch from thrombus resolution to thrombus organisation.

The fibrinogen molecule comprises three pairs of polypeptide chains $(A \alpha, B \beta$ and $\gamma)$, which are linked by disulphide bonds and arranged into three regions: a central (E) region, containing fibrinopeptides $\mathrm{A}$ and $\mathrm{B}$ and the amino acid termini of all six chains; and two distal (D) regions, each formed by the unpaired carboxyl termini of the $\mathrm{A} \alpha, \mathrm{B} \beta$ and $\gamma$ chains [26]. D regions also include a globular $\mathrm{C} \alpha$ domain, formed by the terminal two-thirds of the $\alpha$-chain. Within a given fibrinogen molecule, the two $\mathrm{C} \alpha$ domains are tethered together in close proximity to the E region [27]. Following activation of the coagulation cascade, thrombin cleaves fibrinopeptides A and B from fibrinogen, producing fibrin monomers and initiating clot formation. Thrombin simultaneously activates Factor XIII, promoting cross-linkage between newly formed fibrin oligomers. As part of this process, $\mathrm{C} \alpha$ domains, now untethered from the E region following cleavage of fibrinopeptide $B$, are encouraged to bind with $\mathrm{C} \alpha$ domains from neighbouring fibrin molecules [28]. The resulting $\alpha-\alpha$ linkage strengthens the association between adjacent protofibrils and stabilises the developing fibrin structure. This permits the formation of a fibrin structure that is stronger, more rigid and more resistant to thrombolysis [29].

The pivotal role of the $\mathrm{C} \alpha$ domain in fibrin polymerisation has previously been highlighted by an in vitro study that demonstrated that monoclonal antibodies directed against this domain inhibit the formation of fibrin polymers from monomers [30]. Recombinant fibrinogen lacking the $\mathrm{C} \alpha$ domain has also been shown to form clot in vitro that is less stiff and more susceptible to thrombolysis than clot formed in the presence of normal fibrinogen [31]. Further information about the role of the $\mathrm{C} \alpha$ domain has also been gained from studying hereditary dysfibrinogenaemias [32]. Dusart syndrome, caused by the substitution of Arg fibrinogen $\mathrm{A} \alpha$ for cysteine at position 554 within, is associated with increased $\alpha-\alpha$ chain linkage, and hence enhanced lateral aggregation of fibrin protofibrils. Fibrin clots from patients with this disease tend to be stiff, impermeable and resistant to thrombolysis in vitro [33, 34]. Clinically, patients suffer recurrent thrombotic complications and are particularly prone to embolic phenomena [35]. Conversely, the dysfibrinogen Caracas II, caused by the substitution of serine for asparagines at position 434 within fibrinogen $\mathrm{A} \alpha$, is associated with reduced $\alpha-\alpha$ chain linkage compared with normal fibrinogen. Clots are characteristically loosely packed in structure, and show normal stiffness but increased permeability [36]. Patients with the disorder are typically asymptomatic and do not suffer any haematological complications [36].

Thr312Ala is a well-recognised polymorphism of the A $\alpha$ gene, and encodes a Thr-to-Ala substitution at residue 312 of the $\mathrm{C} \alpha$ domain [37]. Although the functional relevance of this polymorphism remains unclear, the area immediately surrounding this site is known to be central to both Factor XIII activation and to Factor XIII-dependent $\alpha$-chain cross-linking and $\alpha 2$-antiplasmin binding [38, 39]. Clinically, homozygosity for the Ala allele has been associated with acute PE disease but not DVT [14]. The presence of the Ala allele, in both heterozygotes and homozygotes, has also been associated with higher post-stroke mortality in subjects with atrial fibrillation but not those in sinus rhythm [13]. It has, therefore, been suggested that the Ala substitution may predispose to embolic phenomena, through the formation of more brittle clot [40]. In the case of DVT, such clot would be more likely to fragment and dislodge as it propagates proximally and comes under the influence of oblique flow from conjoining veins. The results of the present study support this hypothesis, and contribute further to the evidence that CTEPH is of embolic aetiology.

Evidence from in vitro studies suggests that, other than simply predisposing patients to embolic phenomena, the $\mathrm{A} \alpha$ Thr312Ala polymorphism may play an additional pathophysiological role in CTEPH. Clot formed from Ala/Ala subjects demonstrates more extensive $\alpha$-chain cross-linking, increased fibre thickness and greater clot stiffness than is seen in Thr/Thr controls [40]. Such clot also shows less permeability to flow, which, in turn, permits less access to fibrinolytic factors in vivo [41]. These properties suggest that the Ala substitution enhances lateral aggregation of fibrin protofibrils and encourages formation of a more tightly packed fibrin structure. Although not specifically measured in these studies, such parameters are typically associated with clot that is more resistant to thrombolysis. Moreover, recent data suggest that fibrin derived from CTEPH patients is slower to lyse than that derived from controls [10]. This combined evidence implies that delayed fibrin degradation, possibly mediated in some by the A $\alpha$ Thr312Ala polymorphism, is a pivotal step in the 
development of organised scar tissue from acute PE material in a subset of patients with CTEPH.

The present study also suggested a difference in the frequency of the Factor V Leiden mutation between CTEPH subjects and controls, although this difference did not reach significance $(p=0.051)$. The Factor V Leiden mutation, characterised by substitution of arginine for glutamic acid at position 506 is resistant to cleavage by activated protein $\mathrm{C}$ (APC) and is, therefore, inactivated more slowly [42]. Additionally, the presence of this mutation interferes with the cofactor activity of Factor V on APC-catalysed inactivation of Factor VIIIa [43]. Both mechanisms promote a hypercoagulable state that has been significantly associated with the risk of developing DVT [44]. Previous small studies have shown that the prevalence of this CTEPH mutation is low and not dissimilar to that of controls $[9,45]$. The present study examines a much larger cohort of patients and suggests a possible association, although the prevalence remains much lower than that found in patients with DVT. Given these data, a role for Factor V Leiden in CTEPH cannot be fully discounted, particularly since Factor VIII, a substrate of Factor V, has already been implicated in the disease [8].

Although the present study represents the largest of its kind in $\mathrm{CTEPH}$, it does have several limitations. The use of "convenience" controls that were not necessarily drawn from the same population as the CTEPH cases may have confounded the study, although the results of the Kolmogorov-Smirnov test suggest that population stratification was not an issue. Furthermore, although there is evidence to support a pathophysiological role of both the fibrinogen A $\alpha$ Thr312Ala and Factor V Leiden mutations in thrombotic disease, it is possible that these mutations themselves are not causal but, instead, in LD with other causal loci. Finally, the relatively small numbers of subjects involved meant that the study may not have been powered sufficiently to detect differences in SNP frequencies between the disease and control groups. The differences that were found could have been chance findings in the group of individuals studied, and thus further studies are needed in order to confirm these results. If these future studies were to include a second control group of subjects with a history of completely resolved acute $\mathrm{PE}$, a better understanding of the mechanisms underlying CTEPH could potentially be gained.

In conclusion, the present study demonstrates an association between both the fibrinogen $A \alpha$ substitution of threonine with alanine at position 312 and Factor V Leiden mutations and chronic thromboembolic pulmonary hypertension that adds to understanding of the disease. The presence of these polymorphisms may not only predispose patients to embolic phenomena but also confer resistance to fibrinolysis that subsequently sets the stage for thrombus organisation.

\section{REFERENCES}

1 Hoeper MM, Mayer E, Simonneau G, Rubin LJ. Chronic thromboembolic pulmonary hypertension. Circulation 2006; 113: 2011-2020.

2 Pengo V, Lensing AW, Prins MH, et al. Incidence of chronic thromboembolic pulmonary hypertension after pulmonary embolism. N Engl J Med 2004; 350: 2257-2264.
3 Lang IM. Chronic thromboembolic pulmonary hypertension - not so rare after all. N Engl J Med 2004; 350: 2236-2238.

4 Suntharalingam J, Machado R, Sharples L, et al. Demographics, BMPR2 status and outcomes in distal chronic thromboembolic pulmonary hypertension. Thorax 2007; 62: 617-622.

5 Makris M, Rosendaal FR, Preston FE. Familial thrombophilia: genetic risk factors and management. J Intern Med Suppl 1997; 740: 9-15.

6 Mateo J, Oliver A, Borrell M, Sala N, Fontcuberta J. Laboratory evaluation and clinical characteristics of 2,132 consecutive unselected patients with venous thromboembolism - results of the Spanish Multicentric Study on Thrombophilia (EMET-Study). Thromb Haemost 1997; 77: 444-451.

7 Lang I, Kerr K. Risk factors for chronic thromboembolic pulmonary hypertension. Proc Am Thorac Soc 2006; 3: 568-570.

8 Bonderman D, Turecek PL, Jakowitsch J, et al. High prevalence of elevated clotting factor VIII in chronic thromboembolic pulmonary hypertension. Thromb Haemost 2003; 90: 372-376.

9 Wolf M, Boyer-Neumann C, Parent F, et al. Thrombotic risk factors in pulmonary hypertension. Eur Respir J 2000; 15: 395-399.

10 Morris TA, Marsh JJ, Chiles PG, Auger WR, Fedullo PF, Woods VL Jr. Fibrin derived from patients with chronic thromboembolic pulmonary hypertension is resistant to lysis. Am J Respir Crit Care Med 2006; 173: 1270-1275.

11 Mansilha A, Araujo F, Severo M, Sampaio SM, Toledo T, Albuquerque R. Genetic polymorphisms and risk of recurrent deep venous thrombosis in young people: prospective cohort study. Eur J Vasc Endovasc Surg 2005; 30: 545-549.

12 Varela ML, Adamczuk YP, Forastiero RR, et al. Major and potential prothrombotic genotypes in a cohort of patients with venous thromboembolism. Thromb Res 2001; 104: 317-324.

13 Carter AM, Catto AJ, Grant PJ. Association of the $\alpha$-fibrinogen Thr312Ala polymorphism with poststroke mortality in subjects with atrial fibrillation. Circulation 1999; 99: 2423-2426.

14 Carter AM, Catto AJ, Kohler HP, Ariens RA, Stickland MH, Grant PJ. $\alpha$-Fibrinogen Thr312Ala polymorphism and venous thromboembolism. Blood 2000; 96: 1177-1179.

15 Francis CW. Factor XIII polymorphisms and venous thromboembolism. Arch Pathol Lab Med 2002; 126: 1391-1393.

16 Francis CW. Plasminogen activator inhibitor-1 levels and polymorphisms. Arch Pathol Lab Med 2002; 126: 1401-1404.

17 Jood K, Ladenvall P, Tjarnlund-Wolf A, et al. Fibrinolytic gene polymorphism and ischemic stroke. Stroke 2005; 36 : 2077-2081.

18 Ladenvall $\mathrm{P}$, Johansson L, Jansson JH, et al. Tissue-type plasminogen activator $-7,351 \mathrm{C} / \mathrm{T}$ enhancer polymorphism is associated with a first myocardial infarction. Thromb Haemost 2002; 87: 105-109.

19 Behague I, Poirier O, Nicaud V, et al. $\beta$-Fibrinogen gene polymorphisms are associated with plasma fibrinogen and coronary artery disease in patients with myocardial infarction. The ECTIM Study. Circulation 1996; 93: 440-449.

20 Martiskainen M, Pohjasvaara T, Mikkelsson J, et al. Fibrinogen gene promoter $-455 \mathrm{~A}$ allele as a risk factor for lacunar stroke. Stroke 2003; 34: 886-891. 
21 Coulden R. State-of-the-art imaging techniques in chronic thromboembolic pulmonary hypertension. Proc Am Thorac Soc 2006; 3: 577-583.

22 Scott EM, Ariens RA, Grant PJ. Genetic and environmental determinants of fibrin structure and function: relevance to clinical disease. Arterioscler Thromb Vasc Biol 2004; 24: 1558-1566.

23 Zhao JH, Curtis D, Sham PC. Model-free analysis and permutation tests for allelic associations. Hum Hered 2000; 50: 133-139.

24 Fedullo PF, Rubin LJ, Kerr KM, Auger WR, Channick RN. The natural history of acute and chronic thromboembolic disease: the search for the missing link. Eur Respir J 2000; 15: 435-437.

25 Egermayer P, Peacock AJ. Is pulmonary embolism a common cause of chronic pulmonary hypertension? Limitations of the embolic hypothesis. Eur Respir J 2000; 15: 440-448.

26 Mosesson MW, Siebenlist KR, Meh DA. The structure and biological features of fibrinogen and fibrin. Ann N Y Acad Sci 2001; 936: 11-30.

27 Weisel JW, Medved L. The structure and function of the $\alpha \mathrm{C}$ domains of fibrinogen. Ann N Y Acad Sci 2001; 936: 312-327.

28 Medved LV, Gorkun OV, Manyakov VF, Belitser VA. The role of fibrinogen $\alpha \mathrm{C}$-domains in the fibrin assembly process. FEBS Lett 1985; 181: 109-112.

29 McDonagh RP Jr, McDonagh J, Duckert F. The influence of fibrin crosslinking on the kinetics of urokinase-induced clot lysis. Br J Haematol 1971; 21: 323-332.

30 Cierniewski CS, Budzynski AZ. Involvement of the $\alpha$ chain in fibrin clot formation. Effect of monoclonal antibodies. Biochemistry 1992; 31: 4248-4253.

31 Collet JP, Moen JL, Veklich YI, et al. The $\alpha \mathrm{C}$ domains of fibrinogen affect the structure of the fibrin clot, its physical properties, and its susceptibility to fibrinolysis. Blood 2005; 106: 3824-3830.

32 Matsuda M, Sugo T. Structure and function of human fibrinogen inferred from dysfibrinogens. Int J Hematol 2002; 76: Suppl. 1, 352-360.

33 Collet JP, Soria J, Mirshahi M, et al. Dusart syndrome: a new concept of the relationship between fibrin clot architecture and fibrin clot degradability: hypofibrinolysis related to an abnormal clot structure. Blood 1993; 82: 2462-2469.
34 Collet JP, Woodhead JL, Soria J, et al. Fibrinogen Dusart: electron microscopy of molecules, fibers and clots, and viscoelastic properties of clots. Biophys J 1996; 70: 500-510.

35 Tarumi T, Martincic D, Thomas A, et al. Familial thrombophilia associated with fibrinogen Paris V: Dusart syndrome. Blood 2000; 96: 1191-1193.

36 Woodhead JL, Nagaswami C, Matsuda M, ArochaPinango CL, Weisel JW. The ultrastructure of fibrinogen Caracas II molecules, fibers, and clots. J Biol Chem 1996; 271: 4946-4953.

37 Baumann RE, Henschen AH. Human fibrinogen polymorphic site analysis by restriction endonuclease digestion and allele-specific polymerase chain reaction amplification: identification of polymorphisms at positions $\mathrm{A} \alpha 312$ and Bß 448. Blood 1993; 82: 2117-2124.

38 Kimura S, Aoki N. Cross-linking site in fibrinogen for $\alpha_{2}$-plasmin inhibitor. J Biol Chem 1986; 261: 15591-15595.

39 Muszbek L, Adany R, Mikkola H. Novel aspects of blood coagulation factor XIII. I. Structure, distribution, activation, and function. Crit Rev Clin Lab Sci 1996; 33: 357-421.

40 Standeven KF, Grant PJ, Carter AM, Scheiner T, Weisel JW, Ariens RA. Functional analysis of the fibrinogen $\mathrm{A} \alpha$ Thr312Ala polymorphism: effects on fibrin structure and function. Circulation 2003; 107: 2326-2330.

41 Curran JM, Fatah-Ardalani K, Tornvall P, Humphries SE, Green FR. A hypothesis to explain the reported association of the $\alpha$-fibrinogen A312 allele with thromboembolic disease. Thromb Haemost 2001; 85: 1122-1123.

42 Bertina RM, Koeleman BP, Koster T, et al. Mutation in blood coagulation factor $\mathrm{V}$ associated with resistance to activated protein C. Nature 1994; 369: 64-67.

43 Thorelli E, Kaufman RJ, Dahlback B. Cleavage of factor V at Arg 506 by activated protein $C$ and the expression of anticoagulant activity of factor V. Blood 1999; 93: 2552-2558.

44 Ridker PM, Hennekens CH, Lindpaintner K, Stampfer MJ, Eisenberg PR, Miletich JP. Mutation in the gene coding for coagulation factor $\mathrm{V}$ and the risk of myocardial infarction, stroke, and venous thrombosis in apparently healthy men. N Engl J Med 1995; 332: 912-917.

45 Lang IM, Klepetko W, Pabinger I. No increased prevalence of the factor $\mathrm{V}$ Leiden mutation in chronic major vessel thromboembolic pulmonary hypertension (CTEPH). Thromb Haemost 1996; 76: 476-477. 